\title{
Ein didaktischer Ansatz zu Robert Musils „Möglichkeits- und Wirklichkeitssinn“6 im universitären Unterricht
}

\section{Claudia SPIRIDON ȘERBU}

Lekt. Dr., Transilvania-Universität Kronstadt/Braşov;

E-Mail: claudia.spiridon@ymail.com

\begin{abstract}
Considering the recent criticism of the competence-oriented concern of the PISA study, the present paper proposes a practical teaching concept that opposes the isolated practice of reading skills. In the era of Post-truth, this contribution presents a lesson plan for Robert Musils's novel "The Man without Qualities", which aims at promoting aesthetic sensibility in the universityGerman courses for advanced levels.
\end{abstract}

Keywords: Literature in the German language courses, aesthetic education, Robert Musil

Seit der letzten PISA-Studie (2018) steht das „desaströse“ Bildungswesen in Rumänien wieder zur Debatte, weil Rumänien um neun Positionen in der Rangordnung der Länder zurückgefallen ist. ${ }^{1}$ Die Länder, deren Schüler hervorragende Ergebnisse im PISA-Test nachweisen konnten, werden in Rumänien als Inbegriff eines erfolgreichenSchulsystems gepriesen, wobei jegliche Kritik an der Outputorientierung des standardisierten Messverfahrens überhaupt nicht infrage kommt. Vergleicht man die Auseinandersetzung in Hinblick auf die PISA-Studie in Deutschland oder in den Vereinigten Staaten, so stellt man fest, dass ein Unterricht, der hauptsächlich nach literarischem

1 PISA 2018. România, rezultat dezastros la teste. Analfabetismul funcțional, la apogeu. In: antena3 vom 3.12.2019, online unter: https:// www.antena3.ro/actualitate/educatie/pisa-2018, (Abruf am 9.02.2020). 
Verstehen und Lernerergebnissen fragt, häufig Kritik auf sich zieht. Spinner ${ }^{2}$, Kammler $^{3}$, Frickel ${ }^{4}$, Kepser $^{5}$ oder Wintersteiner ${ }^{6}$ widerlegen die normative Ausrichtung literarischer Bildungsziele, denn „der Irritationscharakter des Ästhetischen“, das "Geltenlassen subjektiverZugänge" erlaube keine Normierung. ${ }^{7}$ Rösch weist darauf hin, dass die durch die Pisa-Studie angeregte Etablierung von Bildungsstandards unter anderem „Ökonomisierung“ zum Nachteil der „Persönlichkeitsbildung“, oder „Können“ zum Nachteil von „Wissen“ als Folge hätte, ${ }^{8}$ und Lösener äußert seine Besorgnis, dass Bildungsstandards das spezifisch Literarische zu stark abschwächen würden. ${ }^{9}$ Der Literaturdidaktiker Johannes Odendahl stellt die These auf, dass im Zeitalter des Post-Faktischen die Menschen mehr als zuvor „den entlastenden, kathartischen Unernst" der Literatur und des Spiels benötigten und suchen werden. ${ }^{10}$ Die politischen Diskurse

2 Spinner, Kaspar H.: Perspektiven ästhetischer Bildung. Zwölf Thesen. In: Vorst, Claudia et al. (Hgg.): Ästhetisches Lernen. Frankfurt 2008, S. 9-23.

3 Kammler, Clemens: Deutschunterricht und Outcome-Orientierung. Zur Kritik eines bildungspolitischen Paradigmas aus fachdidaktischer Sicht. In: Schule vermessen. Friedrich Jahresheft XXX / 2012, S. 23-25.

${ }^{4}$ Frickel, Daniela A./ Kammler, Clemens/ Rupp, Gerhard (Hgg.): Literaturdidaktik im Zeichen von Kompetenzorientierung und Empirie. Perspektiven und Probleme. Freiburg i.Br. 2012.

5 Kepser, Matthis: Anmerkungen zur Kompetenzorientierung in der Literaturdidaktik. In: Frickel et al. 2012, S. 67-84.

6 Wintersteiner, Werner: Wir sind, was wir tun. Poetisches Verstehen als fachdidaktische Herausforderung. In: Winkler, Iris et al. (Hgg.): Poetisches Verstehen. Baltmannsweiler 2010, S. 23-36.

7 Spinner 2008: S. 22.

${ }^{8}$ Rösch, Heidi: Deutschunterricht in der Migrationsgesellschaft. Eine Einführung. Stuttgart 2017, S. 10-11.

9 Lösener, Hans: Die Bildungsstandards und das literarische Lernen. In: Didaktik Deutsch, 36/2014, S. 17-19.

${ }^{10}$ Odendahl, Johannes: Ästhetische Erziehung in Zeiten des Postfaktischen. Zur Legitimation literarischen Lernens. In: LILI-Zeitschrift 
der letzten Jahre zeigen, dass sogar in der Politik erfundene Geschichten auf das Publikum anziehend wirken und weder durch die Berufung auf Fakten noch durch den Bezug auf logische Argumente ihre Attraktivität verlieren. ${ }^{11}$ Laut Odendahl käme der Literatur die Rolle zu, das ästhetische Empfinden anzusprechen und das Grundbedürfnis nach Fiktion zu stillen. Wenn der Literaturunterricht nicht die Flucht aus der Welt der Zwecke und Notwendigkeiten ermöglichte, würden die Lernenden das dort suchen, wo Fiktionen unangebracht sind, wie es das Beispiel der Politik bekundet. ${ }^{12}$

Im Sinne der jüngsten Forderungen nach ästhetischer Erziehung schlage ich im Folgenden ein unterrichtspraktisches Konzept vor, das sich gegen die Entwicklung reiner Lesekompetenzen wendet und ein Kapitel des Romans Robert Musils Der Mann ohne Eigenschaften zum Gegenstand einer 120-minütigen Unterrichtseinheit hat. Es handelt sich um das Romanfragment Wenn es Wirklichkeitssinn gibt, тиß es auch Möglichkeitssinn geben, das zwei Seiten umfasst und dadurch den zeitlichen Rahmen einer Unterrichtseinheit nicht sprengt.

Das hier angeführte Unterrichtskonzept wurde in einer DaF-Lerngruppe, die das Niveau $\mathrm{C} 1$ anstrebte, an der Transilvania-Universität mit einer Gruppe von fünf Studenten durchgeführt. Die hier vorgeschlagenen Aufgaben eignen sich aber auch für das Germanistikstudium, können ergänzt oder in einer veränderten Form eingesetzt werden. Auch wenn auf den ersten Blick die Auswahl des Romans als Textvorlage für den DaF-Unterricht als literarisch sehr anspruchsvoll erscheint, soll der vorliegende Beitrag aufzeigen, dass die Bearbeitung einzelner Auszüge und Themenschwerpunkte den komplizierten Stoff des musilschen Werks sogar für DaF-Lernende zugänglich

für Literaturwissenschaft und Linguistik 47 Jg., 2017, S. 413-428, hier zit. S. 426.

${ }^{11}$ Kittlitz, Alard: Die Erde ist eine Scheibe. In: DIE ZEIT vom 28. August 2016. online unter: www.diezeit.de (Abruf am 12.06. 2019).

${ }^{12}$ Odendahl 2017: S. 427. 
macht. Kaum ein anderer Roman regt in einem so hohen $\mathrm{Ma} ß \mathrm{zu}$ kreativen und spielerischen Schreib- und Sprachaufgaben an, wie Musils Mann ohne Eigenschaften. In der Didaktik-Forschung zum DaF- und DaZ-Unterricht findet Musils Hauptwerk wenig Erwähnung. Es haben sich zwar einige Literaturdidaktiker damit beschäftigt, aber nur unter einzelnen Themenschwerpunkten wie „der andere Zustand“ oder die ironische Darstellung der k.u.k Monarchie. ${ }^{13}$ Dröscher-Teille erwähnte zwar einen Ansatz für die Themenbereiche „Möglichkeitssinn“ und „Eigenschaftslosigkeit", aber sie ging darauf nicht unterrichtspraktisch ein. Neben dem Seitenumfang, der in wenigen Unterrichtseinheiten schwer fassbar ist, begründete Dröscher-Teille Musils marginale didaktische Rezeption dadurch, dass dessen Schrift das Erzählen (discours) zum Nachteil von Handlung bzw. von Geschichte (historie)in den Vordergrund rückt. ${ }^{14}$ Aber gerade das Fehlen eines linearen Handlungsverlaufs erlaubt, unterschiedliche Kapitel des Romans vereinzelt zu didaktisieren, gemischte Textausschnitte im Unterricht einzusetzen, ohne das Verstehen der Kernaussage zu beeinträchtigen.

Robert Musils Spiel mit Begriffen und Sprache, mit den vielfältigen Ausdrucksformen der Realität oder seine nicht präzise Weltauffassung erweitern die Vorstellungskraft der Kursteilnehmer und eröffnen Räume der Schwärmerei. Der Roman bietet alternative Zugänge zu Themen wie Wirklichkeit, Möglichkeit, Leben, Sprache und lässt unterschiedliche Interpretationen zu. Die Hinterfragung von Begriffen wie Ordnung, Kausalität oder Logik, die Musils Romanstruktur grundlegend prägen, kann im Unterricht fruchtbare Diskussionen entzünden und die Lernenden zur Gestaltung neuer Handlungs- und Ausdrucksmöglichkeiten provozieren.

${ }^{13}$ Dröscher-Teille, Mandy: Robert Musil - Schulische Rezeption. In: Nübel, Birgit/ Norbert Christian Wolf(Hgg.): Robert Musil Handbuch. Berlin 2016, S. 866-877, hier zit. S. 871.

${ }^{14}$ Dröscher-Teille 2006: S. 867. 


\section{Methodisch-didaktische Vorüberlegungen}

Die Gestaltung der Unterrichtsstunde fußte methodisch auf Kaspar Spinners handlungs- und produktionsorientierter Literaturdidaktik ${ }^{15}$, wobei Spinners Elf Aspekte des literarischen Lernens ${ }^{16}$ als Bezugspunkte für die Konzeption der Lerntätigkeiten gewählt wurden. Spinner erweiterte die in den 1980er Jahren aufgeführten Prinzipien des handlungs- und produktionsorientierten Literaturunterrichts durch einen rezeptionsästhetischen Ansatz. Für Spinner bedeutet das Lesen nicht nur Informationsentnahme, sondern eine Aufforderung zur Mitwirkung. Vor diesem Hintergrund wurden die Lernaktivitäten so gestaltet, dass dem unterrichtenden Lehrer nicht die Rolle des Vermittlers zukam, sondern er wurde bloß als Teilnehmer an den literarischen Auseinandersetzungen verstanden. Die Interaktion von Text und Lesern konnte so unmittelbar das ästhetische Empfinden der Lernenden ansprechen und sie konnten ihr literaturhistorisches Bewusstsein entwickeln.

Bei den methodisch didaktischen Vorüberlegungen zur Gestaltung des Unterrichts standen folgende Fragen im Vordergrund: welche Lösungen bietet der Text, die auf das eigene Leben des Lernenden anzuwenden sind? Sind Musils essayistische Reflexionen heute noch aktuell? Könnten die Studenten die Möglichkeit wahrnehmen, ihre eigenen Realitätsvoraussetzungen neu zu definieren? Musils Kritik an der Kausalitätsherrschaft und Musils essayistische Haltung der Wirklichkeit gegenüber wurden in Bezug zu den lebensweltlichen Erfahrungen der Lernenden behandelt. Die Wahrnehmung der fiktionalen Welt, die der Lesestoff entfaltet, erfolgte aus der persönlichen

${ }^{15}$ Spinner, Kaspar: Methoden des Literaturunterrichts. In: Kämper van den Boogart, Michael/Kaspar Spinner (Hgg.): Lese- und Literaturunterricht. Kompetenzen und Unterrichtsziele. Methoden und Unterrichtsmaterialien. Baltmannsweiler 2010, S. 190-242.

${ }^{16}$ Spinner, Kaspar: Literarisches Lernen. In: Praxis Deutsch 2/ 2006, S. 6-16. 
Perspektive der Teilnehmenden und förderte dadurch ihr schöpferisches Potenzial.

\section{Etappen der Unterrichtsgestaltung}

Bei der hier angeführten Didaktisierung wurden operative Verfahren in die Einstiegs- und Erarbeitungsphase eingebaut. Das Romanfragment wurde den Lernenden nicht einfach gegeben, um analysiert zu werden, sondern die Aktivitäten wurden so gestaltet, dass sie es ermöglichten, einzelne Sinngehalte des Textes handlungsorientiert zu erschließen. Das operative Umgehen mit dem Text aktivierte dasVorwissen der Studenten und begünstigte das Vermitteln und das Klären neuer Informationen. Ich habe den Einsatz von textproduktiven Verfahren in der Anwendungsphase für sinnvoll erachtet, weil diese Aufgabenstellung Reflexionen über die Sinnzusammenhänge des Textes einstellte und die Auswertung der Ergebnisse ermöglichte.

\section{Operative Verfahren}

Als Einstieg in die Unterrichtsstunde führte ich ein Mindmap zu den Begriffen „Ich“, „Körper“ und „Dinge“ durch, eine Methode mit der die Lernenden vertraut sind. Die Aufgabenstellung lautete:

„1) Erstellen Sie ein Mindmap zu folgenden Begriffen: Ich, Körper, Dinge. Verknüpfen Sie eventuelle Zusammenhänge.“ Mindmaps sprechen sowohl Logik und Analysevermögen als auch Intuition und Vorstellungskraft an. Sie bringen das kreative Potenzial ans Licht und machen gleichzeitig die Komplexität der Lerninhalte besser abrufbar. Beziehungen und Assoziationen werden schnell erstellt, sodass die Studenten weniger Zeit brauchen, um die Informationsflutzubewältigen. ${ }^{17}$ Die Strukturskizze,

${ }^{17}$ Beyer, Maria: Brainland-Mind-Mapping in Aktion. 3. Aufl., Paderborn 2002. 
die im Unterricht entstand,lenkte die Aufmerksamkeit auf zentrale Begriffe wie,,Geist“, „Wahrnehmung“, „Empfindung“, „Eigenschaft“ aber auch „Schmerz“, „Hormone“ oder „Krankheit“. „Dinge“" wurden meistens mit Objekten, die sich im unmittelbaren Umfeld der Lernenden befanden, verknüpft, wie zum Beispiel „Laptop“, „Buch“, „Stuhl“" usw. Die Gedankenlandkarte verdeutlichte Interdependenzverhältnisse zwischen den meisten Elementen, sodass sich am Ende die Frage entpuppte: „Wie wirklich ist eigentlich unsere Wirklichkeit?“. Dass die Lernenden den Wahrheitsgehalt der sie umgebenden Tatsachen anzuzweifeln begannen, war ein Schritt vorwärts zum Verstehendes des musilschen Textfragments.

Beim nächsten Arbeitsschritt wurde zunächst das Kapitel zur Lektüre ausgeteilt, ohne davor Hintergrundinformationen zur Biografie des Autors oder zum historisch-kulturellen Kontext zu klären. Es fand nur eine Vorentlastung hinsichtlich schwieriger oder präsumptiv unbekannter Vokabeln statt. Die Lernenden wurden aufgefordert, den Text individuell zu rezipieren und erste Leseeindrücke aufzuschreiben. Bewertungen wie „eine völlig anstrengende Lektüre“, „,schwierig zu verstehen“, ,interpretationswürdig" tauchten dabei immer wieder auf.

„2) Lesen Sie das Textfragment und notieren Sie erste Leseeindrücke.“

Nach dem Lesen wurden die Studenten in Vierer-Gruppen und eine Dreier-Gruppe eingeteilt. Üblicherweise bevorzuge ich das Durchmischen der Teilnehmer durch die Puzzle-Methode, weil auf diese Weise neue Erfahrungen und neue Kontakte entstehen. Jede Gruppe bekam einen Umschlag, in dem sich unterschiedliche Termini befanden, die die Lernenden den zwei Oberbegriffen „Möglichkeit“ und „Wirklichkeit“" zuschreiben sollten. 
„3) Ordnen Sie die Wörter den zwei Oberbegriffen „Möglichkeit“/,Wirklichkeit" zu.“

Die Kursteilnehmer verknüpften „Subjektivität“, „Perspektive“, „Wahrscheinlichkeit“, ,erproben“, ,,irreal“" oder „experimentieren" mit „Möglichkeit", wobei sie Kennwörter wie „Objektivität“, „Logik“, „Sicherheit“ dem Oberbegriff „Wirklichkeit" zuschrieben. Bei Musil erkennt man Ernst Machs Prinzip der Auflösung des „Ichs“, des „Körpers“, der „Dinge“ und „Kausalverbindungen“. Der österreichische Autor gestaltet ein Weltbild, das auf dem Prinzip des „Möglichkeitssinns“ basiert und definiert diesen ,,als die Fähigkeit [...] alles, was ebensogut sein könnte, zu denken, und das, was ist, nicht wichtiger zu nehmen, als das, was nicht ist" ${ }^{18}$

Die Problematik Wirklichkeit-Möglichkeit führte bei den Studenten zu einer lebhaften Diskussion. Auch wenn die zwei abstrakten Begriffe am Anfang Schwierigkeiten bereitet hatten, erhöhte sich im Rahmen des Meinungsaustausches die Beteiligung am Unterrichtsgespräch und die Zahl der spontanen Einfälle. Im Verlauf der Diskussion haben sich manche Lernende auf die Seite des Möglichkeitsmenschen positioniert, andere haben sich für die Lebensform des Wirklichkeitsmenschen eingesetzt. Es war interessant zu beobachten, dass sie die Position, die sie zu rechtfertigen versuchten, als ihre eigene Lebenseinstellung anpriesen, sie nahmen die Innenperspektive eines „Möglichkeitsmenschen“ beziehungsweise eines „Wirklichkeitsmenschen" an und begannen allmählich Nachteile und Vorteile für jede der zwei Wesensarten zu suchen. Die „Möglichkeitsmenschen“ dehnten den Begriff der „Möglichkeit" in die Richtung einer neu gestalteten Lebensform aus. Sie gaben an, dass vor dem Hintergrund zahlreicher technologischen Herausforderungen, denen das Individuum des einundzwanzigsten Jahrhunderts täglich begegne, die moderneWirklichkeit nicht mehr durch Logik und Sinn erfasst werden könne. Die

${ }^{18}$ Musil, Robert: Der Mann ohne Eigenschaften. 17. Aufl. Bonn 2003, S. 16 . 
Teilnehmer behaupteten, dass der Begriff der „Möglichkeit“ die Flucht aus einem von Desillusionierung und Bedeutungslosigkeit geprägten Alltag ermögliche und erlaube, neue Lebensbedingungen zu schaffen, die die Phantasie lebendig erhalten. Der „Möglichkeitsmensch“ sollte seinen Sinn auf Veränderung richten und die Wirklichkeit als eine „Hypothese“ betrachten. Die „Wirklichkeitsmenschen“ beriefen sich dagegen auf Sicherheit und Logik und wiesen die Risiken, auf die sich eventuell ein „Möglichkeitsmensch“ einlassen würde, ab. Sie koppelten den „Möglichkeitssinn“ mit Zweifel und Irrtum und sprachen sich für eine „Wirklichkeit“ aus, die unmittelbar an konkrete Erfahrungen gebunden ist. Das Wesen eines „Wirklichkeitsmenschen"führten sieals von Besonnenheit, Selbstbeherrschung und Gleichgewicht geprägt an.

Im Anschluss an die Diskussionsrunde orientierten sich die nächsten Lerntätigkeiten an Musils Konstruktion der Möglichkeiten und koppelten dabei die fiktionale Ebene des Textes mit den persönlichen Erfahrungen der Studenten. Musils Ansatzpunkte für eine hypothetische Wirklichkeit wurden in der Fachliteratur mit Friedrich Nietzsches philosophischen Grundideen in Zusammenhang gebracht. Sowohl Musil als auch Nietzsche bezeichneten den „Möglichkeitsmenschen“ als einen, für den es keine objektive Wahrheit gebe, sondern nur Interpretationen dessen, was für einen „Wirklichkeitsmenschen“ ein Geflecht von Ursache und Wirkung sein könnte. ${ }^{19}$

\section{Textproduktive Verfahren}

Spinner deutet darauf hin, dass die Kreativität der Lernenden durch textproduktive Verfahren stärker als durch operative gefördert wird, da die produktive Herangehensweise lebendigere

${ }^{19}$ Hinz, Michael: Verfallsanalyse und Utopie. Nietzsche-Rezeption in Thomas Manns „,Zauberberg“ und in Robert Musils „,Mann ohne Eigenschaften “. St. Ingbert 2000, S. 132-157. 
Auseinandersetzungen mit dem literarischen Text erlaubt. ${ }^{20}$ Schreibaufgaben ermöglichen den Lernenden, die vorgegebenen Textvorlagen kreativ umzuwandeln und mit ihrem eigenen Erfahrungshorizont zu konfrontieren. Als Aufgabe sollten sie das Online-Profil des „Möglichkeitsmenschen“ oder des „Wirklichkeitsmenschen" erstellen und Musils komplizierten Satzbau in eine spielerische Jugendsprache umwandeln.

„4) Erstellen sie das Online-Profil des Möglichkeits- oder des Wirklichkeitsmenschen. Sie können auch Bilder benützen.“

Musil selbst schuf in seinem Roman einen „Möglichkeitsmenschen" und übertrug auf dessen Handlungen, auf dessen Gedanken und Entscheidungen die Eigenart des „Möglichkeitssinnes". Musils Hauptfigur Ulrich versucht sein in Konventionen gestaltetes Dasein durch das „Möglichkeitsdenken“ zu überwinden und die Wirklichkeit in Hinblick auf die Möglichkeit zu überschreiten. Die Art, in der die Lernenden das Online-Profil des „Möglichkeitsmenschen“bzw. „Wirklichkeitsmenschen“ aufbauten und strukturierten, gab ihre individuelle Stellungnahme zum Text wieder. Sie konnten ihre Deutungshypothesen konkret überprüfen und ihre eigene Interpretationsweise verlautbaren. Der breite Spielraum von Innen- und Außenperspektiven stellte ihnen zahlreiche Wege zur Identitätsfindung und zum Fremdverstehen zur Verfügung.

Am Ende der Unterrichtseinheit führte ich eine weitere Aktivität ein, die den Einfallsreichtum und das Denken der Teilnehmenden stimulierte. Musils philosophische Grundgedanken zum Themenkomplex „Möglichkeitssinn“-, ,Wirklichkeitssinn" sollten diesmal als Rollenspiel umgesetzt werden. Den Ausgangspunkt bildete ein Konflikt zwischen zwei Partnern, die sich bei der Wahl des Urlaubsziels nicht einigen können. Jeder Partner sollte seine Argumente für oder gegen ein Urlaubsziel im Sinne des „Möglichkeitsmenschen“ bzw.

${ }^{20}$ Spinner 2010: S. 252. 
„Wirklichkeitsmenschen“ konstruieren, was zur Perspektivenübernahme als auch zum Perspektivenwechsel anregte.

„5) Sie planen mit ihrem Partner eine Reise für den Sommer. Sie können sich aber nicht für Ihr Reiseziel entscheiden und streiten. Verfassen Sie einen Dialog, in dem Sie ihre Argumentation im Sinne des Möglichkeits- bzw. Wirklichkeitsmenschen konstruieren."

Der Themenkomplex „Wirklichkeitssinn“-,Möglichkeitssinn" hat sich in der Gestaltung der Figuren, die am Dialog beteiligt waren, in der Auswahl der Charaktereigenschaften, die die Lernenden mit den Gestalten verknüpft haben und in den Variationen des Sprachcodex widerspiegelt. Das Vorstellungsvermögen war vor allem dadurch gefragt, dass die Teilnehmer Musils philosophischen Grundgedanken auf die Gegenwart bezogen und eine lebensnahe Beobachterposition einnahmen. Die Umsetzung der reflexiven Ideenkette in Form eines Dialogs, der eine gewisse Dynamik und eine Spannung aufwies, vertiefte das Textverständnis und führte gleichzeitig zur Intensivierung des Lernprozesses. Die Personen transportierten Musils Weltanschauung in die Gegenwart und waren an die technologisierten Voraussetzungen der Jetztzeit gebunden. Das Rollenspiel begünstigte die Kommunikation zwischen Leser und Text, denn die Teilnehmenden wurden wechselnden Gesprächskonstellationen ausgesetzt und dadurch gezwungen, die zwei gegensätzlichen philosophischen Auffassungen differenziert zu betrachten. Die Auffassung vom Möglichkeits- und Wirklichkeitssinn, die die Studenten in der ersten operativen Phase des Unterrichts durchschaut hatten, wurde während des Schreibprozesses neu relativiert. Der Deutungsinhalt des Textes wurde neu entfaltet, die Lernenden haben selbst durch Montage neue Sinngehalte aktiv entdeckt und gebaut. Die spielerische Darstellung von realitätsnahen Situationen ermöglichte es den Teilnehmenden sich von den Zwängen des Alltags zu distanzieren, sich selbst 
und die Mitspieler zu beobachten und sich auf eine Welt des Zusammenspiels von Realität und Fiktionen einzulassen.

\section{Schlussbemerkungen}

Robert Musils Roman ist im Zeitalter des Postfaktischen aktueller als je zuvor. Auch wenn die Auseinandersetzung mit diesem Roman im heutigen DaF-Unterricht zunächst obsolet erscheinen mag, liegt in Robert Musils Weltanschauung ein Moment der Modernität für unsere Zeit, sodass unterschiedliche Fragmente seines Romans im DaF-Unterricht lesenswert sind.

Die ästhetische Erziehung sollte im schulischen und universitären Bereich in Rumänien zu den Hauptanliegen in der Konzeption der Unterrichtseinheit gehören, denn die Entwicklung der emotionalen Seite ist genauso wichtig wie die Förderung kognitiver Fähigkeiten. Literatur im DaF-Unterricht soll mehr als nur Informationsaustausch sein und einen praktisches Nutzen haben, sie soll den Lernenden die Flucht in eine irreale Welt ermöglichen. Die hier vorgestellten Lerntätigkeiten im Zusammenhang mit Musils Roman wenden sich gegen die zweckrationale Ausrichtung des Unterrichts und verschaffen dem Ästhetischen mehr Raum. Sie wenden sich gegen eine bloße Vermittlung von Lerninhalten und gegen das isolierte Einüben von Fähigkeiten, indem sie Denken und Empfinden gleichzeitig fördern. Tatsächlich sind die unterschiedlichen Facetten des Themenkreises Möglichkeitssinn-Wirklichkeitssinn in einer Diagnose der Jahrhundertwende fundiert, jedoch hat dieser Themenkomplex die Studenten zu lebhaften Diskussionen angeregt. Die offene Struktur des Textfragments rief die Lust am Spiel und an abstrakten Gedankenkonstruktionen hervor und bot den Lernenden persönliche Anknüpfungspunkte und vielfältige Umsetzungsmöglichkeiten. Die unmittelbaren Auswirkungen, die das Textfragment auf die Lernenden hatte, konnten nicht gleich ermessen werden. Inwieweit sich ihr ästhetisches 
Bewusstsein verändert hat, und ob sie die gewonnenen Einsichten in ihr Alltagsleben integrieren werden, sind Fragen, die erst mit der Zeit beantwortet werden können. Am Ende der Unterrichtseinheit konnte man nur bemerken, dass die Lernenden ihre interpretativen Fähigkeiten, ihre Vorstellungskraft, Kreativität und Empathie entwickelt haben und nach dem Unterricht das Gespräch weiterführten.

\section{Literaturverzeichnis}

\section{Primärliteratur}

Musil, Robert: Tagebücher. Hrsg. von Adolf Frisé, Hamburg 1976.

\section{Sekundärliteratur}

Beyer, Maria: Brainland - Mind-Mapping in Aktion. 3. Aufl., Paderborn 2002.

Dröscher-Teille, Mandy: Robert Musil - Schulische Rezeption. In: Nübel, Birgit/ Norbert Christian. Wolf (Hgg.): Robert Musil Handbuch. Berlin 2016, S. 866-877.

Frickel, Daniela A./ Kammler, Clemens/ Rupp, Gerhard (Hgg.):

Literaturdidaktik im Zeichen von Kompetenzorientierung und Empirie. Perspektiven und Probleme. Freiburg i.Br. 2012.

Hinz, Michael: Verfallsanalyse und Utopie. Nietzsche-Rezeption in Thomas Manns ,Zauberberg “ und in Robert Musils „Mann ohne Eigenschaften“.St. Ingbert 2000.

Kammler, Clemens: Deutschunterricht und Outcome-Orientierung. Zur Kritik eines bildungspolitischen Paradigmas aus fachdidaktischer Sicht. In: Schule vermessen. Friedrich Jahresheft XXX/ 2012, S. 23-25.

Kepser, Matthis: Anmerkungen zur Kompetenzorientierung in der Literaturdidaktik. In: Frickel et al. 2012, S. 67-84. 
Lösener, Hans: Die Bildungsstandards und das literarische Lernen. In: Didaktik Deutsch, 36/2014, S. 17-19.

Odendahl, Johannes: Ästhetische Erziehung in Zeiten des Postfaktischen. Zur Legitimation literarischen Lernens. In: LiLi-Zeitschrift für Literaturwissenschaft und Linguistik, 47 Jg. 2017, S. 413-428, oder unter: https://link.springer. com/article/10.1007/s41244-017-0066-2 (Abruf am 12. 06. 2019).

PISA-Konsortium (Hg.): PISA 2000 - Die Länder der Bundesrepublik Deutschland im Vergleich. Opladen 2002, S. 55-95.

Rösch, Heidi: Deutschunterricht in der Migrationsgesellschaft. Eine Einführung. Stuttgart 2017, S. 10-11.

Spinner, Kaspar: Literarisches Lernen. In: Praxis Deutsch33/ 2006, S. 6-16.

Spinner, Kaspar H.: Perspektiven ästhetischer Bildung. Zwölf Thesen. In: Vorst, Claudia et al. (Hgg.): Ästhetisches Lernen. Frankfurt 2008, S. 9-23.

Spinner, Kaspar: Methoden des Literaturunterrichts. In: Kämper van den Boogart, Michael/Kaspar Spinner (Hgg.): Lese- und Literaturunterricht. Kompetenzen und Unterrichtsziele. Methoden und Unterrichtsmaterialien. Baltmannsweiler 2010, S. 190-242.

Wintersteiner, Werner: Wir sind, was wir tun. Poetisches Verstehen als fachdidaktische Herausforderung. In: Winkler, Iris et al. (Hgg.): Poetisches Verstehen. Baltmannsweiler 2010, S. 23-36.

\section{Internetquellen}

Kittlitz, Alard: Die Erde ist eine Scheibe. In: DIE ZEIT vom 28. August 2016, online unter: https://www.zeit.de/2016/ 36/luegen-politik-donald-trump-rudy-giuliani-thomas-demaiziere-gefuehlte-wahrheit (Abruf am 12. 06. 2019). 